\title{
OPEN Genetic diversity and population structure analysis of Lateolabrax maculatus from Chinese coastal waters using polymorphic microsatellite markers
}

Wei Wang ${ }^{1}$, Chunyan $\mathrm{Ma}^{1}$, Longling Ouyang ${ }^{2}$, Wei Chen ${ }^{1}$, Ming Zhao ${ }^{1}$, Fengying Zhang ${ }^{1}$, Yin Fu ${ }^{1}$, Keji Jiang ${ }^{1}$, Zhiqiang Liu ${ }^{1}$, Heng Zhang ${ }^{2}$ \& Lingbo Ma ${ }^{1 凶}$

In order to provide valuable guidelines for the conservation of germplasm of Lateolabrax maculatus, the genetic diversity and population structure analysis were evaluated for eight geographic populations along coastal regions of China, using 11 microsatellite DNA markers. The genetic parameters obtained showed that, eight populations can be clustered into two groups, the Northern group and the Southern group, concordant with their geographical positions. The UPGMA tree constructed according to the Nei's genetic distance along with the structure analysis and discriminant analysis of principal component also supported this result. This might be explained by the geographic separation and the divergent environmental conditions among the populations. It's worth noting that, $Q D$ (Qingdao) population from northern area was assigned to the Southern group and showed a close genetic relationship and similar genetic constitution with the southern populations. We speculated that large scales of anthropogenic transportation of wild fries from QD populations to the southern aquaculture areas in history should be the primary cause. The populations from GY (Ganyu), $\mathrm{RD}$ (Rudong) and BH (Binhai) had higher genetic diversity and showed limited genetic exchange with other populations, indicating better conservation of the natural resources in these regions. All populations were indicated to have experienced bottleneck events in history.

Chinese sea bass, Lateolabrax maculatus, is an economically important species for Asian countries. In China, it is a primary aquaculture marine fish widely distributed along the coastal areas. However, the long-term over-fishing of both fry and adult fish, habitat destruction, water pollution, and other factors resulted in dramatic decline of their wild resources. The wild fries were almost extinct around the Yangtze estuary ${ }^{1}$. Moreover, introgression of the breeding offspring and adult fish escaped from culture ponds or cages also negatively impacted on the biodiversity of the wild resources of $L$. maculatus, similar to what has been proved in Salmo salar ${ }^{2,3}$. Therefore, there was an urgent need to protect the L. maculatus wild resources in China with scientific approaches.

It is widely accepted that the knowledge of the genetic studies of wild stocks can be performed using molecular markers such as mtDNA, microsatellite loci and isoenzyme. Among these, microsatellites have provided very useful data comparing the genetic diversity between wild and cultured populations of marine species, aiding in the conservation management of over-exploited populations and the corresponding policy design ${ }^{4-6}$. Although several genetic studies of this species had been reported in China, they were mainly focused on L. maculatus populations in partial areas, such as Shandong Peninsula, and the sample sizes analyzed were relatively small. Besides, most of these studies were outdated (before 2010) ${ }^{1,7-13}$. The genetic diversity and population structure of L. maculatus might have changed over the past ten years. One recent study in 2017 showed, a lower nucleotide diversity and a higher haplotype diversity indicating L. maculatus in China has experienced potential population expansion. However, a significant divergence was only found between Qingdao and Fangcheng populations based on mitochondrial COI gene sequences from all five populations analyzed ${ }^{14}$. Another study of six geographic

${ }^{1}$ Key Lab of Marine and Estuarine Fisheries Resources and Ecology, Ministry of Agriculture, East China Sea Fisheries Research Institute, Chinese Academy of Fishery Sciences, No. 300 Jungong Road, Yangpu District, Shanghai 200090, China. 'East China Sea Fisheries Research Institute, Chinese Academy of Fishery Sciences, Shanghai 200090, China. ${ }^{\circledR}$ email: malingbo@vip.sina.com 


\begin{tabular}{|l|l|l|l|l|l|l|l|l|l|l|l|}
\hline Locus & $\mathbf{N a}$ & $\mathrm{Ne}$ & $\mathrm{Ho}$ & $\mathrm{He}$ & $\mathbf{P I C}$ & $\mathbf{I}$ & HWE & $\mathbf{P}_{\mathbf{N}}$ & Fis & Fit & Fst \\
\hline Lama 04 & 23.0000 & 4.2220 & 0.5238 & 0.7644 & 0.7417 & 1.9064 & 0.0000 & 0.0694 & 0.1966 & 0.3088 & 0.1396 \\
\hline Lama 10 & 23.0000 & 5.8124 & 0.4302 & 0.8295 & 0.8130 & 2.2182 & 0.0000 & 0.1965 & 0.4368 & 0.4870 & 0.0892 \\
\hline Lama 21 & 29.0000 & 10.5124 & 0.8299 & 0.9068 & 0.8979 & 2.6765 & 0.9841 & 0.0131 & 0.0009 & 0.1001 & 0.0993 \\
\hline Lama 23 & 32.0000 & 7.8937 & 0.7979 & 0.8748 & 0.8606 & 2.4129 & 0.9999 & 0.0231 & 0.0295 & 0.0987 & 0.0713 \\
\hline Lama 24 & 40.0000 & 6.5374 & 0.6860 & 0.8485 & 0.8351 & 2.4619 & 1.0000 & 0.0364 & 0.0611 & 0.1967 & 0.1445 \\
\hline Lama 28 & 8.0000 & 1.8610 & 0.3049 & 0.4637 & 0.4328 & 0.9473 & 0.0000 & 0.1135 & 0.3571 & 0.6683 & 0.4841 \\
\hline Lama 31 & 44.0000 & 19.7277 & 0.8900 & 0.9509 & 0.9470 & 3.2455 & 1.0000 & 0.0123 & 0.0354 & 0.0673 & 0.0331 \\
\hline Lama 32 & 25.0000 & 5.5269 & 0.5461 & 0.8205 & 0.8048 & 2.2308 & 0.0000 & 0.0727 & 0.2127 & 0.3355 & 0.1560 \\
\hline Lama 36 & 17.0000 & 6.278 & 0.7082 & 0.8422 & 0.8219 & 2.0793 & 0.0000 & 0.0437 & 0.0983 & 0.1728 & 0.0826 \\
\hline Lama 38 & 23.0000 & 5.864 & 0.5119 & 0.8309 & 0.8104 & 2.1341 & 0.0000 & 0.0957 & 0.2472 & 0.3795 & 0.1757 \\
\hline Lama 44 & 52.0000 & 16.4509 & 0.8908 & 0.9408 & 0.9360 & 3.2095 & 1.0000 & 0.0136 & 0.0213 & 0.0525 & 0.0318 \\
\hline Mean & 28.7273 & 8.2442 & 0.6473 & 0.8248 & 0.8092 & 2.3202 & & 0.0627 & 0.1325 & 0.2362 & 0.1196 \\
\hline
\end{tabular}

Table 1. Genetic diversity of 11 microsatellite loci in eight $L$. maculatus populations. Na observed number of alleles, $\mathrm{Ne}$ expected number of alleles, $\mathrm{Ho}$ observed heterozygosity, $\mathrm{He}$ expected heterozygosity, PIC polymorphic information content, $I$ shannon wiener index, $H W E$ deviations from Hardy-Weinberg equilibrium, $P_{N}$ frequency of null alleles, Fis inbreeding coefficient, Fit total in-coefficient of population.

\begin{tabular}{|l|l|l|l|l|l|l|}
\hline & Na & Ne & I & Ho & He & PIC \\
\hline QD & 10.0000 & 4.8794 & 1.6515 & 0.6289 & 0.7068 & 0.6742 \\
\hline FC & 10.5455 & 5.6762 & 1.7896 & 0.6470 & 0.7580 & 0.7219 \\
\hline DT & 8.5455 & 4.5958 & 1.6216 & 0.6131 & 0.7270 & 0.6822 \\
\hline LY & 9.4545 & 4.4636 & 1.5253 & 0.6441 & 0.6640 & 0.6273 \\
\hline CM & 10.0909 & 4.7693 & 1.6582 & 0.6133 & 0.7115 & 0.6782 \\
\hline GY & 18.0000 & 10.3263 & 2.4847 & 0.7728 & 0.9120 & 0.8730 \\
\hline RD & 11.6364 & 6.1103 & 1.8850 & 0.6016 & 0.7896 & 0.7501 \\
\hline BH & 15.3636 & 8.3063 & 2.2311 & 0.6813 & 0.8573 & 0.7153 \\
\hline
\end{tabular}

Table 2. Genetic diversity statistics of eight L. maculatus populations. LY Lieyu, CM Chongming, DT Dongtou, QD Qingdao, FC Fangcheng, GY Ganyu, RD Rudong, BH Binhai, Na observed number of alleles, Ne expected number of alleles; I shannon wiener index, $H o$ observed heterozygosity, $H e$ expected heterozygosity, PIC polymorphic information content.

populations of L. maculatus suggested that this species could be divided into two groups, i.e. Southern and Northern group according their geographic locations ${ }^{15}$. Apparently, these results are insufficient to provide a comprehensive understanding of current genetic background of wild L. maculatus resources in China. There is renewed interest in updating the genetic information of this species.

In our study, the genetic diversity within and among the eight wild L. maculatus geographic populations collected from the coastal regions in China and their population structures were investigated using 11 microsatellite DNA markers. It is expected that this study can largely enrich the genetic information of L. maculatus flocks in China, which can be used for not only the protection of its wild resources, but also for improving the developing sustainable fishing management policy.

\section{Results}

Genetic diversity. The genetic diversity indices of 11 microsatellite loci in eight L. maculatus populations are shown in Table 1. In total, 316 alleles were detected in 294 individuals, with an average value of 28.7273 alleles per loci. The highest Ne (expected number of alleles) (19.7277) value was found in Lama 31 locus, while the Lama 28 locus exhibited the lowest number of Ne (1.861). The PIC (polymorphic information content) values lay in the range from 0.4328 (Lama 28) to 0.9470 (Lama 31) with a mean value of 0.8092 . All loci showed a high polymorphic (PIC $>0.5)$ except Lama 28 with a medium value $(0.5>$ PIC $>0.25)$. The Fis (inbreeding coefficient) value per locus averaged 0.1325, ranging from 0.0009 (Lama 21) to 0.4368 (Lama 10), while the Fit (total incoefficient of population) value was 0.2362 averagely. Both MICROCHECKER and FreeNA revealed evidence for presence of null alleles in all loci. However, as null allele frequencies for each locus computed by EM algorithm were all much less than 0.2 (ranged from 0.0131 to 0.1135 ) (Table 1), and each L. maculatus populations analyzed in present study consisted of at least 25 individuals, the existence of null alleles was considered not to affect the results of following genetic analysis $6,16,17$.

The genetic diversity parameters of eight L. maculatus populations based on 11 microsatellite markers are listed in Table 2. The results indicated that GY population had the highest genetic diversity among eight 


\begin{tabular}{|l|l|l|l|l|l|l|l|l|}
\hline & QD & FC & DT & LY & CM & GY & RD & BH \\
\hline QD & 0.0525 & 22.4773 & 7.7245 & 2.1360 & 3.5264 & 1.4995 & 1.2719 & 1.7142 \\
\hline FC & $0.0110^{*}$ & 0.1416 & 5.5721 & 1.9113 & 2.7884 & 1.7354 & 1.3882 & 1.9315 \\
\hline DT & $0.0314^{*}$ & $0.0429^{*}$ & 0.1430 & 4.1873 & 13.4262 & 1.7900 & 1.4277 & 2.1109 \\
\hline LY & $0.1048^{*}$ & $0.1157^{*}$ & $0.0563^{*}$ & 0.0362 & 14.5604 & 1.4565 & 1.0998 & 1.6676 \\
\hline CM & $0.0662^{*}$ & $0.0823^{*}$ & $0.0183^{*}$ & $0.0169^{*}$ & 0.1000 & 1.6723 & 1.3370 & 1.9186 \\
\hline GY & $0.1429^{*}$ & $0.1260^{*}$ & $0.1226^{*}$ & $0.1465^{*}$ & $0.1301^{*}$ & 0.0892 & 3.1514 & 9.6471 \\
\hline RD & $0.1643^{*}$ & $0.1526^{*}$ & $0.1490^{*}$ & $0.1852^{*}$ & $0.1575^{*}$ & $0.0735^{*}$ & 0.2271 & 5.0032 \\
\hline BH & $0.1273^{*}$ & $0.1146^{*}$ & $0.1059^{*}$ & $0.1304^{*}$ & $0.1153^{*}$ & $0.0253^{*}$ & $0.0476^{*}$ & 0.1955 \\
\hline
\end{tabular}

Table 3. Genetic differentiation coefficient (Fst, below diagonal), gene flow (Nm, above diagonal) and inbreeding coefficient (Fis, on diagonal) for eight L. maculatus populations. LY Lieyu, CM Chongming, DT Dongtou, $Q D$ Qingdao, FC Fangcheng, GY Ganyu, $R D$ Rudong, $B H$ Binhai ${ }^{\star} P<0.01$.

\begin{tabular}{|l|l|l|l|l|l|l|l|l|}
\hline & QD & FC & DT & LY & CM & GY & RD & BH \\
\hline QD & $* * * *$ & 0.9035 & 0.8835 & 0.7565 & 0.8290 & 0.1968 & 0.3239 & 0.2891 \\
\hline FC & 0.1015 & $* * * *$ & 0.8032 & 0.6572 & 0.7251 & 0.1919 & 0.3028 & 0.3042 \\
\hline DT & 0.1239 & 0.2191 & $* * *$ & 0.8592 & 0.9195 & 0.2539 & 0.3515 & 0.3280 \\
\hline LY & 0.2791 & 0.4197 & 0.1518 & $* * *$ & 0.9494 & 0.3289 & 0.3701 & 0.3910 \\
\hline CM & 0.1876 & 0.3215 & 0.0839 & 0.0519 & $* * * *$ & 0.2960 & 0.3816 & 0.3660 \\
\hline GY & 1.6255 & 1.6506 & 1.3708 & 1.1119 & 1.2173 & $* * * *$ & 0.4037 & 0.4881 \\
\hline RD & 1.1272 & 1.1946 & 1.0455 & 0.9940 & 0.9633 & 0.9070 & $* * * *$ & 0.7643 \\
\hline BH & 1.2409 & 1.1899 & 1.1146 & 0.9391 & 1.0052 & 0.7173 & 0.2689 & $* * * *$ \\
\hline
\end{tabular}

Table 4. Nei's genetic identity (above diagonal) and genetic distance (below diagonal) for eight L. maculatus populations. LY Lieyu, CM Chongming, DT Dongtou, QD Qingdao, FC Fangcheng, GY Ganyu, RD Rudong, $B H$ Binhai.

populations, while the lowest genetic parameter values were mostly observed in LY population. In general, GY, $\mathrm{BH}$ and $\mathrm{RD}$ (Rudong population) had the higher genetic diversity comparing to other populations.

Genetic differentiation and population structure. The pair-wise Fst (genetic differentiation coefficient values) and Nm (gene flow) value among eight L. maculatus populations are shown in Table 3 . The Fst values between each two populations ranged from 0.0110 to 0.1852 , and the genetic differentiation between each two populations reached a significant level $(P<0.01)$. The highest Fst value was observed between RD and LY populations, while the lowest value was found for FC (Fangcheng) and QD (Qingdao) populations. GY, RD and $\mathrm{BH}$ populations showed high level differentiation when compared to the other five populations. These results suggested that all populations could be divided into two groups, GY, RD and BH could be classified into one group, while the remaining five populations were assigned to the other group. Consistent with the results of genetic differentiation, eight populations could be clustered into two groups based on pair-wise Nm values. The gene flow between GY, RD, $\mathrm{BH}$ and other five populations $(\mathrm{Nm}<2.2)$ were much lower than that between each two of those three populations $(\mathrm{Nm}>3.1)$.

Consistent with the results of genetic differentiation and gene flow, GY, RD and BH populations showed large genetic distance and low genetic identity when compared to the other five populations (Table 4). As a result, all eight populations are grouped into two main genealogical branches in Fig. 1. RD and BH populations converged first, then gathered with GY population and separated from other populations. The remaining five populations formed the other branch. Among them, QD and FC clustered as a group. In the other group, DT population was separated, while LY and CM populations clustered as a small branch, which represented a closer relationship between the two.

An analysis of molecular variance (AMOVA) test was performed in order to evaluate the genetic diversity among and within populations. As presented in Table 5, 89.86\% of total variations were found among populations, and $10.14 \%$ were observed within populations. The fixation index was 0.10140 (Table 5).

The mutation-drift equilibrium tests were performed for eight L. maculatus populations in this study. As shown in Table 6, none of all populations deviate from equilibrium under IAM and TPM models in Sign Test $(P>0.05)$, except for $\mathrm{BH}$. However, all populations showed a high percent of heterozygous deficiency under SMM model, and significantly deviated from mutation-drift equilibrium $(P<0.01)$. This can be supported the positive value of Fis obtained in present study (Table 3). In Wilcoxon Sign-rank Test, BH population showed no deviation from mutation-drift equilibrium under all three models $(P>0.05)$. In contrast, QD, FC, LY and CM populations deviated from equilibrium with extreme significance $(P<0.01)$, while DT, GY and RD population deviated from 


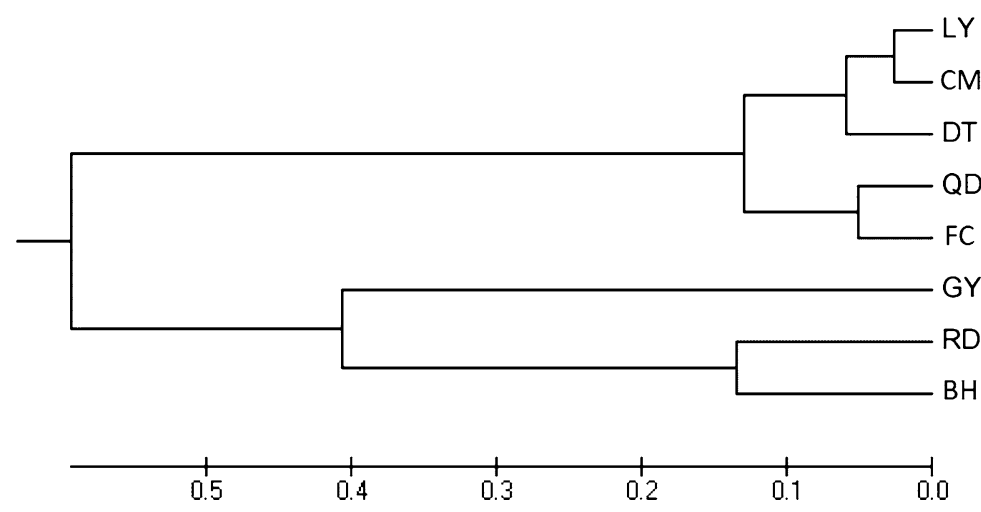

Figure 1. UPGMA clustering tree of L. maculatus populations based on Nei's distance. LY Lieyu, CM Chongming, DT Dongtou, QD Qingdao, FC Fangcheng, GY Ganyu, RD Rudong, BH Binhai.

\begin{tabular}{|l|r|l|l|l|l|l|}
\hline Source of variation & \multicolumn{1}{|c|}{ d.f } & Sum of squares & Variance components & Percentage of variation & Fst & P \\
\hline Among populations & 7 & 198.889 & $0.34648 \mathrm{Va}$ & 10.14 & 0.10140 & 0.00000 \\
\hline Within populations & 580 & 1780.956 & $3.07061 \mathrm{Vb}$ & 89.86 & & \\
\hline Total & 587 & 1979.845 & 3.4171 & & & \\
\hline
\end{tabular}

Table 5. AMOVA results for eight L. maculatus populations.

\begin{tabular}{|c|c|c|c|c|c|c|c|c|c|}
\hline & \multicolumn{6}{|c|}{ Sign test } & \multicolumn{3}{|c|}{ Wilcoxon Sign-rank test } \\
\hline & \multicolumn{2}{|l|}{ IAM } & \multicolumn{2}{|l|}{ TPM } & \multicolumn{2}{|l|}{ SMM } & \multirow{2}{*}{\begin{tabular}{|l|} 
IAM \\
$P$ \\
\end{tabular}} & \multirow{2}{*}{\begin{tabular}{|l|} 
TPM \\
$P$ \\
\end{tabular}} & \multirow{2}{*}{\begin{tabular}{|l|} 
SMM \\
$P$
\end{tabular}} \\
\hline & $\mathrm{He} / \mathrm{Hd}$ & $P$ & $\mathrm{He} / \mathrm{Hd}$ & $P$ & $\mathrm{He} / \mathrm{Hd}$ & $P$ & & & \\
\hline QD & $7 / 4$ & 0.53554 & $4 / 7$ & 0.09504 & $1 / 10$ & $0.00104^{* *}$ & 0.46484 & 0.14746 & $0.00244^{* *}$ \\
\hline FC & $9 / 2$ & 0.11644 & $6 / 5$ & 0.47553 & $1 / 10$ & $0.00096^{\star *}$ & $0.01221^{*}$ & 0.96582 & $0.00684^{\star *}$ \\
\hline DT & $6 / 5$ & 0.46898 & $5 / 6$ & 0.24571 & $2 / 9$ & $0.00688^{*}$ & 0.32031 & 0.27832 & $0.01611^{\star}$ \\
\hline LY & $7 / 4$ & 0.50164 & $6 / 5$ & 0.47852 & $1 / 10$ & $0.00085^{\star *}$ & 0.70020 & 0.46484 & $0.00098^{* *}$ \\
\hline $\mathrm{CM}$ & $7 / 4$ & 0.52851 & $4 / 7$ & 0.11366 & $0 / 11$ & $0.00005^{\star *}$ & 0.17480 & 0.14746 & $0.00049^{* *}$ \\
\hline GY & $8 / 2$ & 0.20461 & $4 / 6$ & 0.10082 & $1 / 9$ & $0.00308^{\star *}$ & 0.13086 & 0.32227 & $0.00488^{*}$ \\
\hline $\mathrm{RD}$ & $9 / 2$ & 0.11578 & $7 / 4$ & 0.50695 & $1 / 10$ & $0.00101^{\star *}$ & 0.12305 & 0.89844 & $0.00342^{* *}$ \\
\hline $\mathrm{BH}$ & $10 / 1$ & $0.03228^{*}$ & $7 / 4$ & 0.53376 & $2 / 9$ & $0.00594^{* *}$ & 0.05371 & 1.00000 & 0.05371 \\
\hline
\end{tabular}

Table 6. Results of mutation-drift equilibrium tests of eight L. maculatus populations. LY Lieyu, CM Chongming, DT Dongtou, QD Qingdao, FC Fangcheng, GY Ganyu, RD Rudong, BH Binhai, He/Hd represents the ratio of heterozygote excess and heterozygous deficiency loci number. ${ }^{\star}$ Means significant deviation from equilibrium $(P<0.05) .{ }^{* \star}$ Means extremely significant deviation from equilibrium $(P<0.01)$.

equilibrium significantly under SMM model $(P<0.05)$. In addition, FC population showed significant deviations under both IAM model $(P<0.05)$ and SMM model $(P<0.01)$.

The proportion of membership for L. maculatus across eight populations was generated under different cluster numbers (K values) by STRUCTURE software (Fig. 2). When K equaled to 2, most individuals from QD, FC, DT, LY and CM populations were assigned to cluster 1, while most individuals of GY, RD and BH populations were assigned to cluster 2 . When $\mathrm{K}$ equaled to 3, individuals were divided into three clusters, with most individuals of QD and FC assigned to cluster 1, and part of QD population assigned to cluster 3. Most individuals from DT, $\mathrm{LY}$ and CM populations were assigned to cluster 3, while most individuals of GY, RD and BH populations were assigned to cluster 2. When K equaled to 4, most individuals of QD, FC, DT, LY and CM populations showed similar genetic structure to that of $\mathrm{K}$ equaled to 3. Most individuals of GY and $\mathrm{BH}$ were assigned to cluster 3, meanwhile most individuals from RD population were assigned to cluster 4. A similar genetic structure among the individuals from eight populations was also obtained by DAPC (Discriminant Analysis of Principal Component) (Fig. 3). 

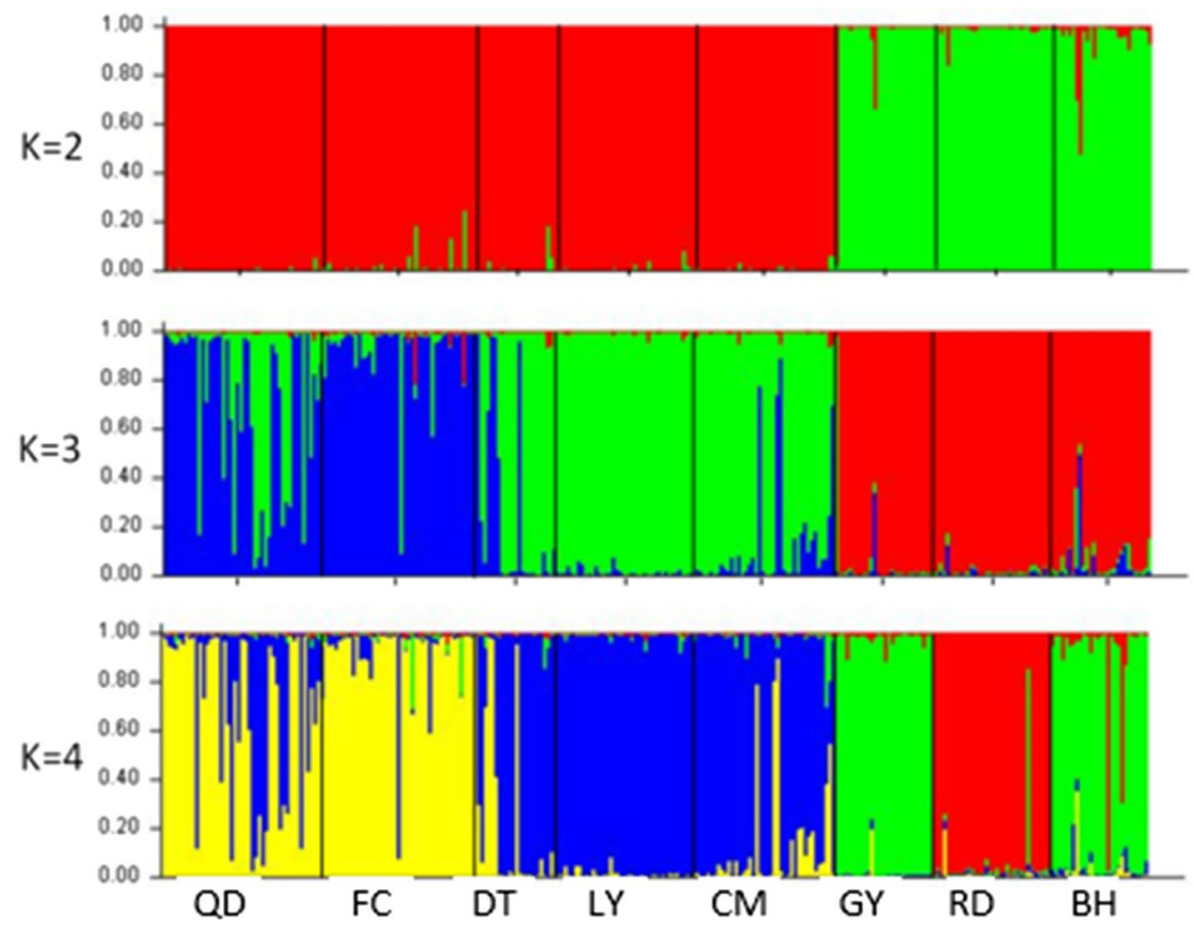

Figure 2. STRUCTURE genetic cluster analysis for L. maculatus populations. LY Lieyu, CM Chongming, DT Dongtou, QD Qingdao, FC Fangcheng, GY Ganyu, RD Rudong, BH Binhai. The population names were given below the box plot with the individuals of different populations separated by vertical black lines. Each color represents "one population".

\section{Discussion}

Polymorphism diversity analysis of microsatellite markers. In the study of population genetics, molecular markers such as isozyme, mtDNA and microsatellites DNA have been widely used to monitor the genetic diversity within and between populations of many fishery species, including L. maculatus ${ }^{12,14,18-21}$. Compared with the other molecular markers, microsatellites have many advantages, including co-dominant inheritance, highly polymorphism and random dispersion in the genome ${ }^{22}$. However, only a few researches of $L$. maculatus applied microsatellites, although a number of microsatellite markers have been developed ${ }^{23-25}$. In our study, 11 microsatellite loci originally described by Shao et $\mathrm{al}^{23}$ were tested. All loci were successfully amplified. The mean value of $\mathrm{Na}(28.7273)$ and PIC (0.8092) for them were both higher than that in former studies of $L$. maculatus based on microsatellite ${ }^{14,23,24}$. Also, 10 of the 11 loci verified in this study present a high level of polymorphism diversity $(\mathrm{PIC}>0.5)^{26}$. These results indicated that the microsatellite loci tested in present study were effective molecular genetic markers and could be used to precisely estimate the genetic diversity of different populations of L. maculatus.

Chi-square tests showed that the six microsatellite loci implied an extremely significant deviation from HWE. Meanwhile, the heterozygote deficiency which can lead to the departure from HWE was also indicated by the positive values of Fis for each locus (Table 1). The departure from HWE could be induced by many factors, such as genetic drift, small population size, null alleles, genetic mutation, non-random mating and Wahlund effect ${ }^{6,27}$. In the present study, the genetic drift can be excluded as the overall large $\mathrm{Nm}$ value $(\mathrm{Nm}>1)$ among populations, the sample size of each population is also bigger than former studies ${ }^{1,7-13}$. Therefore, it might result from the high mutation rate of the specific nucleotide in the sequence targeted by the primer, which can lead to failure of PCR amplification and the detection of null alleles in this study (Table 1$)^{28}$. Similar results could also be found in earlier studies ${ }^{15,23,24,29-31}$, which indicates this was a common phenomenon when microsatellite markers are used in population genetic researches. Meanwhile, the Wahlund effect cannot be ruled out as overall Fst (0.1196) is close to mean Fis $(0.1325)$ (Table 1$)^{6}$. In addition, as shown in Fig. 2, stratification within population leading to the existence of subpopulations could also contribute to the deviation from HWE in this study ${ }^{23}$.

Population genetic diversity. In our study, the overall genetic characteristics identified were similar to that in earlier researches ${ }^{1,15,23,29}$, and higher than that assessed by mtDNA markers ${ }^{14}$. It is suggested that these 11 microsatellite loci were sufficient to evaluate the genetic information in the present study. In addition, the genetic characteristics of $\mathrm{BH}$ population sampled from the Bohai Sea were higher than that reported by Shao et $\mathrm{al}^{23}$. The higher value of genetic parameters in this study might result from the larger number of L. maculatus individuals used. As the allele number and the mutation rate at each polymorphic locus are positively correlated with to the sample size ${ }^{29}$. Furthermore, owing to the high resolution of capillary electrophoresis labeled by fluo- 


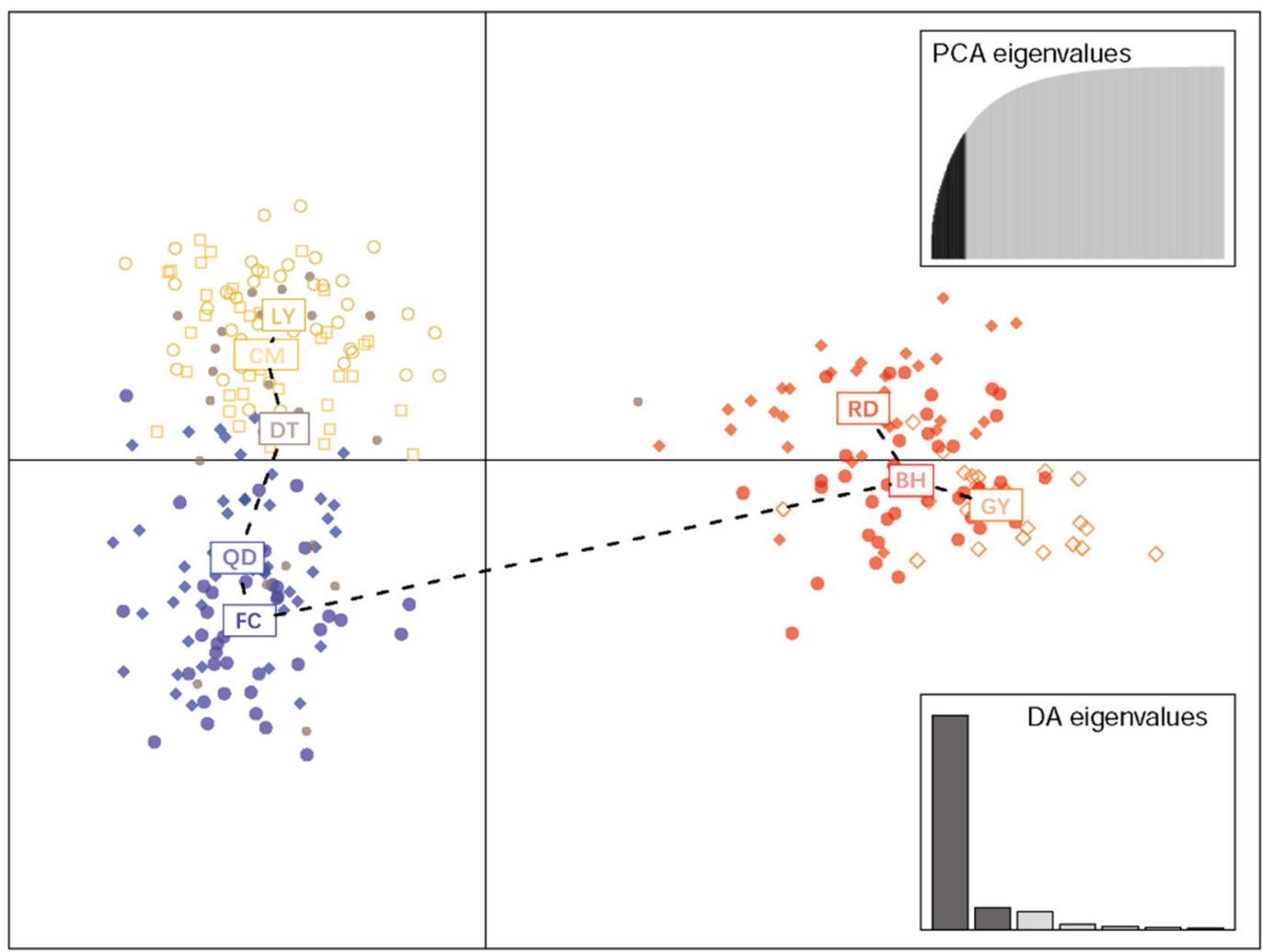

Figure 3. DAPC plot diagram of L. maculatus populations. LY Lieyu, CM Chongming, DT Dongtou, $Q D$ Qingdao, FC Fangcheng, GY Ganyu, RD Rudong, $B H$ Binhai. Colored dots with different shapes represent individuals from different geographical populations, PCA and DA scatterplots on the right side of the graph indicate the principal components and numbers of discriminant functions for the computations.

rescent markers, the genetic diversity parameters were obtained with higher value and accuracy compared with traditional methods ${ }^{31}$.

According to the PIC values, all eight $L$. maculatus populations showed a high genetic diversity $(\mathrm{PIC}>0.5)^{26}$ and could be arranged in the following order: $\mathrm{GY}>\mathrm{BH}>\mathrm{RD}>\mathrm{FC}>\mathrm{CM}>\mathrm{QD}>\mathrm{DT}>\mathrm{LY}$. This is consistent with the notion that the genetic resource from the northern areas of China is better than that from the southern areas $^{12,15,32,33}$. However, the genetic diversity of QD population from the northern area was much lower in the present study. These is because that $L$. maculatus individuals from northern coastal regions of China are widely acknowledged to have better environmental adaptability and growth performance ${ }^{1,10,32,33}$. Therefore, large number of wild individuals were captured and transported to southern aquaculture areas of China from the $1990 \mathrm{~s}^{1}$. The situation was particularly serious in QD, due to its convenient geographic traffic environment. Correspondingly, the population structure of this fish in Shandong Peninsula changed from 2000 to $2006^{1}$. In contrast, the other northern populations such as GY, BH and RD populations showed a better genetic conservation. For the L. maculatus populations clustered to southern group, FC population showed the highest genetic variability, followed by $\mathrm{CM}$ population. It is easier to understand the relatively higher genetic diversity of CM population, because it is located in estuary area of Yangtze River. These coastal waters were considered to be the natural breeding areas for aquatic animals due to the favorable environmental conditions and sufficient food supply ${ }^{31}$. The frequent gene exchange with other locations as indicated by the high $\mathrm{Nm}$ and low Fis values also played an important part (Table 3). As for FC population, its particularly high genetic diversity might derive from the strong genetic conservation due to the geographic isolation formed by Leizhou Peninsula ${ }^{13,14}$, and the supplement of germplasm resources from the northern region, which can be supported by its close genetic relationship with QD population.

Genetic differentiation and genetic structure. In our study, the differentiations among eight populations all reached a significant level $(P<0.01)$. However, in a previous genetic analysis of five L. maculatus populations based on mitochondrial COI gene, four of ten pairwise comparisons indicated insignificant genetic differentiation ${ }^{14}$. This is because mitochondrial DNA markers are maternal inheritance and can be easily influenced by selective pressure. They are not sensitive enough when used to analyze the genetic structure and gene flow of populations located in a small geographical area. In contrast, microsatellite markers used in this study can identify the weak genetic differentiation due to serveral advantages, such as high polymorphism, co-dominant inheritance, wide distribution, abundance and rapid evolutionary rate re $^{34}$.

Eight L. maculatus populations in this study could be divided into two groups based on Fst value. QD, FC, DT, LY and CM populations clustered as one group, while GY, RD and $\mathrm{BH}$ populations formed the other group. 
Overall, the genetic differentiation was found at a low or medium level within each group, whereas a medium or high level was observed between them ${ }^{35}$. These results provide new evidence for the conclusion that the $L$. maculatus populations along China coastal regions has experienced significant genetic divergence, and differentiated into the northern and southern groups ${ }^{1,12-14}$. It can also be verified by the results of genetic distance, genetic identity, UPGMA tree, STRUCTURE genetic cluster analysis and DAPC in our study (Table 4, Figs. 1, 2 and 3). As the gene flow among eight population were strong enough $(\mathrm{Nm}>1)$ to prevent the genetic differentiation resulted from genetic drift ${ }^{36}$. The geographic segregation, ocean currents and habit differences such as the lower water temperature in the northern area which can limit the dispersal capacity of fish, might result in the divergence of those two groups. It's worth noting that QD population was assigned to the southern group genetically, although it belongs to the northern group geographically. A similar result was also reported in previous study ${ }^{14}$. As mentioned above, the large scale of anthropogenic transportation of wild L. maculatus individuals from QD to the southern aquaculture areas of China in history should be dominant reason ${ }^{1}$. It can be proven by the highest value of Nm indicating the sufficient gene exchange between QD and FC populations (Table 3). Consequently, QD population showed a much closer genetic relationship with and similar genetic component to the southern populations. With regard to the other southern populations including DT, LY and CM, the convenient gene exchange between them should account for the clustering and their similar genetic component, as there was no obvious geographic barrier among them.

When $\mathrm{K}$ value equaled to $4, \mathrm{RD}$ population was separated from $\mathrm{BH}$ and GY populations. This separation might result from the following reasons: Firstly, the lower water temperature in the northern regions of China coastal regions limited the migration capacity of L. maculatus. Secondly, the geographic barrier formed by Shandong Peninsula might separate BH population from GY and RD populations. Thirdly, the invisible ocean current might result in the difference in genetic structure between RD and GY populations $s^{37}$, as their geographical distance is relatively small and no obvious barriers exists between these two locations. According to the STRU CTURE analysis, RD population showed nearly no gene mixture with other populations and exhibited an unique genetic structure, accounting for the high genetic differentiation between RD and other populations (Table 3). Moreover, a high genetic diversity was also detected in $\mathrm{RD}$ population. All these results suggested the genetic resource of wild L. maculatus in this region was well conserved. It's worth noting that, although the genetic diversity was higher in the northern group, their larger overall Fis value especially in RD population, indicated a possibility of future population depression, as the lower water temperature has limited their gene exchange with other populations (Table 3). Therefore, the protection of breeding group in these populations and the gene exchange with other populations should be reinforced.

Demographic bottleneck. According to bottleneck analysis, these L. maculatus populations might have experienced a recently consecutive genetic bottleneck. As for short time bottleneck events, they can only influence the abundance of alleles but not their frequency. In contrast, continuous bottleneck effects can both result in the change of genetic variants and decline of genetic diversity ${ }^{38-40}$. Correspondingly, QD, FC, LY and CM populations in our study showed a lower genetic diversity compared with the other four populations. In previous studies, Weihai, Beihai, as well as QD L. maculatus populations were confirmed to have encountered bottlenecks ${ }^{1,14}$. It was also reported that all three populations of L. japonicus in Korea had experienced bottleneck events, because the overfishing in history and degradation of the environment has led to a decline in the sea bass population $^{41}$. The L. maculatus populations in China were also under a similar situation. In order to meet the increasing market demands, a large number of wild fish fries were captured and utilized in the artificial culture activities of L. maculatus in history. As a result, there was a long-term overfishing pressure for the germplasm resources of this species, and ecological environment of its habitats was also destroyed by the fast-growing aquaculture industry. These changes in the recent decades may contribute to the bottlenecks observed in our study. In our previous study, the L. maculatus populations in China was suggested to have experienced potential population expansion events after a period of small effective population size ${ }^{14}$. These results emphasized the importance of protecting in the germplasm resources of L. maculatus and its habitats.

As the solution, artificial breeding of the seedlings of L. maculatus can mitigate the overfishing pressure of wild fries, and the protection of marine environment and strict management of closed fishing seasons will aid in restoration of germplasm resources. Furthermore, the germplasm bank of wild L. maculatus from different areas of China should be well constructed.

\section{Materials and methods}

Ethics declaration. All sampling fish were not endangered or protected species. In China, catching wild $L$. maculatus from sea waters does not require specific permits. Our study was approved by the ECSFRI (East China Sea Fisheries Research Institute, China Academy of Fisheries Science, Shanghai, China), and the study was carried out in compliance with the ARRIVE guidelines. All experiments were performed according to national law and guidelines of the animal care and use policies set by ECSFRI.

Samples collection and DNA extraction. A total of 294 wild L. maculatus individuals were collected along the coastal regions of China, including Binhai of Tianjin City (BH, N=38), Qingdao of Shangdong Province (QD, N =46), Ganyu, Rudong of Jiangsu Province (GY, N=28, RD, N=34), Chongming of Shanghai City $(\mathrm{CM}, \mathrm{N}=43$ ), Dongtou of Zhejiang Province (DT, $N=25)$, Lieyu of Fujian Province (LY, $\mathrm{N}=40$ ) and Fangcheng of Guangxi Province (FC, $\mathrm{N}=40$ ) (Fig. 4). The sample size of each population in this study is similar or larger than that in previous related researches ${ }^{1,15,23}$, which is enough to provide scientific results. Dorsal muscle was sampled from each fish and genomic DNA was extracted using TIANamp Marine Animals Genomic DNA 


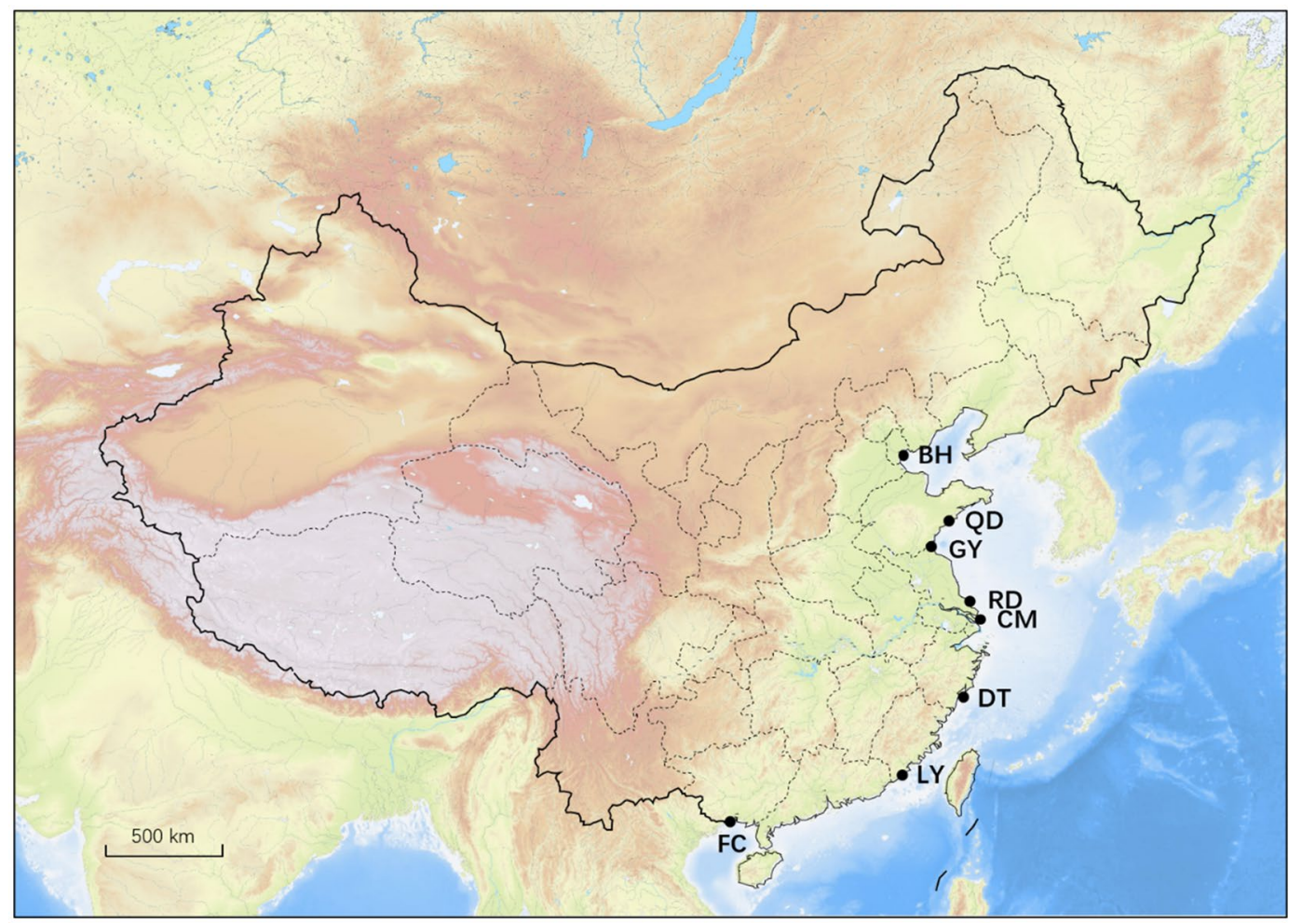

Figure 4. Geographical locations of the eight L. maculatus populations (generated by software BIGEMAP version 29.1.4.0, URL link: http://www.bigemap.com/). BH Binhai, QD Qingdao, GY Ganyu, RD Rudong, $C M$ Chongming, DT Dongtou, LY Lieyu, FC Fangcheng.

\begin{tabular}{|c|c|c|c|c|}
\hline Locus name & Primer sequences $\left(5^{\prime}-3^{\prime}\right)$ & Repeat motif & Temperature $\left({ }^{\circ} \mathrm{C}\right)$ & Allele size (bp) \\
\hline \multirow{2}{*}{ Lama 04} & F: TTGTTGTAAGTAGTGGTGGGAAT & \multirow{2}{*}{$(\mathrm{AC}) 9$} & \multirow{2}{*}{54} & \multirow{2}{*}{$172-176$} \\
\hline & R: AAAAATGAAGGAGGACAGAATGA & & & \\
\hline \multirow{2}{*}{ Lama 10} & F: CAGACACACCCGAAAGAAAGT & \multirow{2}{*}{$(\mathrm{CA}) 9$} & \multirow{2}{*}{54} & \multirow{2}{*}{$224-230$} \\
\hline & R: CTGAAGTTACCTGTCTCAAGCA & & & \\
\hline \multirow{2}{*}{ Lama 21} & F: TCCAGGTCTGTTTTCTGTTTC & \multirow{2}{*}{$(\mathrm{AC}) 13$} & \multirow{2}{*}{54} & \multirow{2}{*}{$242-260$} \\
\hline & R: TTTTCTCGGATTATCTGTCTCA & & & \\
\hline \multirow{2}{*}{ Lama 23} & F: AACTGACGGAGATGATACGGT & \multirow{2}{*}{$(\mathrm{GT}) 15$} & \multirow{2}{*}{54} & \multirow{2}{*}{$148-154$} \\
\hline & R: GCTGAAGAAGAGGCAGGTGT & & & \\
\hline \multirow{2}{*}{ Lama 24} & F: TCCCAAATCGTCTTGTCGGC & \multirow{2}{*}{$(\mathrm{GT}) 8$} & \multirow{2}{*}{54} & \multirow{2}{*}{$160-170$} \\
\hline & R: CACACGCTGTTCACATTCTGCA & & & \\
\hline \multirow{2}{*}{ Lama 28} & F: AATCACGCAGAAAGTGGAAA & \multirow{2}{*}{$(\mathrm{CA}) 10$} & \multirow{2}{*}{54} & \multirow{2}{*}{$154-160$} \\
\hline & R: AGACAGATGGGACGCATAAAC & & & \\
\hline \multirow{2}{*}{ Lama 31} & F: CCAGGGGGCAGACAGGAGGT & \multirow{2}{*}{ (TG)13 } & \multirow{2}{*}{54} & \multirow{2}{*}{$216-248$} \\
\hline & R: GCCCCATTCTTCCTCCAACCA & & & \\
\hline \multirow{2}{*}{ Lama 32} & F: GTGCTGGTGCCTAAACGAACG & \multirow{2}{*}{$(\mathrm{CA}) 15$} & \multirow{2}{*}{54} & \multirow{2}{*}{$200-212$} \\
\hline & R: TTTCCTGTGCTGCCTGGTGA & & & \\
\hline \multirow{2}{*}{ Lama 36} & F: CTAAAGGACCACAAGATACACG & \multirow{2}{*}{$(\mathrm{AC}) 13$} & \multirow{2}{*}{54} & 2760 \\
\hline & R: ACTCAGGCTCAAACCAGACA & & & $276-280$ \\
\hline J 38 & F: ACAAAACTCATCCATCAAGCAG & (CT) 1 & 50 & 10 204 \\
\hline Lama 38 & R: AGTGTCCACGGAGACGGTAA & $(G 1) 11$ & 54 & $204-210$ \\
\hline Lama 44 & F: GGGCAGTAATTGGTGAGGGA & (GT & 5 & $148+3$ \\
\hline Lama 44 & R: TCTTCAGGGCAAAAGGTGGT & $(G 1) 16$ & 54 & $148-160$ \\
\hline
\end{tabular}

Table 7. Characterization of L. maculatus microsatellite primers. 
Extraction Kit (TIANGEN, DP324-03, China) according to manufacture protocols. The extracted DNAs were stored at $-20^{\circ} \mathrm{C}$ until use.

Microsatellite amplification. In this study, 11 specific microsatellite loci for L. maculatus as previously described were amplified after test ${ }^{23}$. The information of 11 microsatellite primers used in the present study was listed in Table 7. The fluorescent primers were synthesized with the 5' end of each forward primer labeled with a FAM or Hex fluorescent tag. PCR amplification was performed in a $20 \mu$ l solution consisting of 0.5 unit of Taq polymerase, $2.0 \mu \mathrm{l} 10 \times$ buffer, $0.5 \mu \mathrm{dNTP}(50 \mathrm{mM}$ each), $1.0 \mu \mathrm{l}$ each primer $(10 \mu \mathrm{M})$, and $1 \mu$ l genomic DNA $(50 \mathrm{ng} / \mu \mathrm{l})$. Then $\mathrm{ddH}_{2} \mathrm{O}$ was added to the PCR mixture to make a final volume of $20 \mu \mathrm{l}$. PCR amplifications were conducted under a PCR touchdown protocol: initial denaturation at $95^{\circ} \mathrm{C}$ for $5 \mathrm{~min}$, followed by 10 cycles of $95^{\circ} \mathrm{C}$ for $30 \mathrm{~s}, 62-52^{\circ} \mathrm{C}$ for $30 \mathrm{~s}$ (reducing $1{ }^{\circ} \mathrm{C}$ for each cycle) and $72{ }^{\circ} \mathrm{C}$ for $30 \mathrm{~s}$ of extension, 25 cycles of denaturation at $95^{\circ} \mathrm{C}$ for $30 \mathrm{~s}$, alignment of $52^{\circ} \mathrm{C}$ for $30 \mathrm{~s}, 72^{\circ} \mathrm{C}$ for $30 \mathrm{~s}$ of extension, followed by a final extension step of $72^{\circ} \mathrm{C}$ for $7 \mathrm{~min}$. After that, the approximate size and concentration of PCR products were analyzed using agarose gel electrophoresis, PCR products were mixed according to non-overlapping fragment size and fluorescence marker (FAM or Hex), and then all samples were detected by capillary electrophoresis in an Applied Biosystem 3730XL DNA Analyzer sequencer, using LIZ 500 ladder as reference.

Data analysis. The accurate product size and genotypes of all samples were analyzed using the software GeneMapper, Ver 3.0 (ABI, USA). All data obtained was imported into Microsoft Excel for further analysis. The presence of PCR errors such as large allele dropout, null alleles were investigated by the software MICOCHECKER ${ }^{6,42}$, allele frequencies $\left(\mathrm{P}_{\mathrm{N}}\right)$ for each locus and population were calculated by the EM algorithm (expectation maximization algorithm) using FreeNA ${ }^{6,43}$ The specific genetic diversity indices including observed number of alleles $(\mathrm{Na})$, expected number of alleles $(\mathrm{Ne})$, observed heterozygosity $(\mathrm{Ho})$, expected heterozygosity (He), Shannon Wiener index (I), inbreeding coefficient (Fis), total in-coefficient of population (Fit) were calculated using POPGEN version 3.2 ${ }^{44}$. Deviations from Hardy-Weinberg equilibrium (HWE) were tested using ARLEQUIN version 3.11, by using the Monte Carlo Markov Chain Method ${ }^{45}$. Polymorphic information content (PIC) was analyzed by PIC_CALV software version $0.6^{46}$. Nei's genetic distance (Ds) and gene flow (Nm) among different $L$. maculatus populations were computed by POPGEN version $3.2^{44}$. An UPGMA phylogenetic tree was constructed by MEGA software 5.0 version, following the Kimura-2-parameter (K2P) distance model based on the Ds values ${ }^{47,48}$. ARLEQUIN (version 3.11) was used to calculate the genetic differentiation coefficient values (Fst) and perform the analysis of molecular variance (AMOVA) ${ }^{45}$. The significance of AMOVA components was analyzed by 1000 permutations. Demographic bottleneck for each of eight L. maculatus populations was analyzed using BOTTLENECK version 3.4, based on Infinite Allele Model (IAM), Two-phased Model of Mutation (TPM) and Step-wise Mutation Model (SMM). The significance of difference excess heterozygosity was evaluated by Sign Test and Wilcoxon Sign-rank Test ${ }^{49}$. STRUCTURE (version 2.3) was employed to carry out a Bayesian clustering analysis. K value was set from 2 to 4 , with a burn in period of 50,000 and a run length of 50,000 . Five replicates were used for each $\mathrm{K}$ value, while the default values were set for the rest of the parameters. The optimum number of clusters among eight populations was also calculated by STRUCTURE software (version 2.3), which could be used to assess the theoretical population number based on the genetic structure of the eight L. maculatus populations ${ }^{50}$. Finally, discriminant analysis of principal component (DAPC) was performed by the ADEGENET in R (version 4.0.2) . $^{51}$

\section{Data availability}

The datasets generated during and/or analyzed during the current study are available from the corresponding author on reasonable request.

Received: 16 January 2021; Accepted: 7 June 2021

Published online: 27 July 2021

\section{References}

1. Jiang, X., Yang, G. P., Wei, Q. W. \& Zou, G. W. Analysis of the genetic structure of spotted sea bass (Lateolabrax maculatus) inhabiting the Chinese Coast. Period. Ocean Univ. China 39, 271-274 (2009) (In Chinese with English abstract).

2. Clifford, S. L., McGinnity, P. \& Ferguson, A. Genetic changes in Atlantic salmon (Salmo salar) populations of northwest Irish rivers resulting from escapes of adult farm salmon. Can. J. Fish. Aquat. Sci. 55, 358-363 (1998).

3. Bourret, V., O’Reilly, P. T., Carr, J. W., Berg, P. R. \& Bernatchez, L. Temporal change in genetic integrity suggests loss of local adaptation in a wild Atlantic salmon (Salmo salar) population following introgression by farmed escapees. Heredity 106, 500-510 (2011).

4. Hutchings, J. A. \& Fraser, D. J. The nature of fisheries- and farming-induced evolution. Mol. Ecol. 17, 294-313 (2008).

5. Reiss, H., Hoarau, G., Dickey-Collas, M. \& Wolff, W. J. Genetic population structure of marine fish: Mismatch between biological and fisheries management units. Fish Fish. 10, 361-395 (2009).

6. Giantsis, I. A., Mucci, N., Randi, E., Abatzopoulos, T. J. \& Apostolidis, A. P. Microsatellite variation of mussels (Mytius galloprovincialis) in Central and Eastern Mediterranean: Genesc panmixia in the Aegean and the Ionian Sea. J. Mar. Biol. Assoc. U.K. 94, 797-809 (2014).

7. Liu, J. X., Gao, T. X., Yokogawa, K. \& Zhang, Y. P. Differential population structuring and demographic history of two closely related fish species, Japanese sea bass (Lateolabrax japonicus) and spotted sea bass (Lateolabrax maculatus) in Northwestern Pacific. Mol. Phylogenet. Evol. 39(3), 799-811 (2006).

8. Hu, Z. M., Gao, T. X., Han, Z. Q. \& Song, L. Studies on genetic differentiation of the spotted sea bass (Lateolabrax maculatus) and Japanese sea bass (Lateolabrax japonicus). Period. Ocean Uni. China 37(3), 413-418 (2007) (In Chinese with English abstract).

9. Nakayama, K., Kineshita, I. \& Seikai, T. Morphologlcal comparisons during early stage rearing of Chinese and Japanese forms of the temperate bass, Lateolabrax japonicus. Jpn. J. Ichthyol. 43(1), 13-20 (1996). 
10. Lou, D., Gao, T. X. \& Zhang, X. M. The advances on germplasm resources study of Lateolabrax. J. Zhejiang Ocean Univ. (Natural Science) 19(2), 162-167 (2000) (Chinese in English abstract).

11. Lou, D. et al. Study on genetic variation in Chinese and Japanese sea bass. Journal of Ocean University of Qingdao 33(1), 22-28 (2003) (In Chinese in English abstract).

12. Li, M. Y., Zhao, M. Z., Zhong, A. H. \& Xue, L. Y. The analysis of genetic variation of Lateolabrax japonicus from Rizhao of Shandong and Xiamen of Fujian by isozyme and RAPD methods. Acta Oceanol. Sin. 27(3), 119-123 (2005) (In Chinese with English abstract).

13. Liu, M. Y., Jiang, Q. C. \& Yang, J. X. Analysis on mitochondrial DNA cytochrome b gene of Lateolabrax japonicas from different seas. J. Nanjing Normal Univ. (Natural Science Edition) 33(1), 102-106 (2010) (In Chinese with English abstract).

14. Wang, W. et al. Population genetic diversity of Chinese sea bass (Lateolabrax maculatus) from southern coastal regions of China based on mitochondrial COI gene sequences. Biochem. Syst. Ecol. 71, 114-140 (2017).

15. Wang, G. X. et al. Genetic variability in six Lateolabrax maculatus populations inhabiting the Chinese coast. J. Fish. Sci. China 24(2), 395-402 (2017) (In Chinese with English abstract).

16. Chapuis, M. P., Loiseau, A., Michalakis, Y., Lecoq, M. \& Estoup, A. Characterization and PCR multiplexing of polymorphic microsatellite loci for the locust Locusta migratoria. Mol. Ecol. Notes 5(3), 554-557 (2005).

17. Chapuis, M. P. \& Estoup, A. Microsatellite null alleles and estimation of population differentiation. Mol. Biol. Evol. 24(3), 621-631 (2007).

18. Tan, Y., Fang, L., Qiu, M., Huo, Z. M. \& Yan, Y. W. Population genetic of the Manila clam (Ruditapes philippinarum) in East Asia. Sci. Rep. 10, 21890 (2020).

19. Agulló, M. et al. Genetic analyses reveal temporal stability and connectivity pattern in blue and red shrimp Aristeus antennatus populations. Sci. Rep. 10, 21505 (2020).

20. An, H. S., Lee, J. W. \& Kim, H. Y. Genetic differences between wild and hatchery populations of Korean spotted sea bass (Lateolabrax maculatus) inferred from microsatellite markers. Genes Genom. 35(5), 671-680 (2013).

21. An, H. S., Kim, H. Y. \& Kim, J. B. Genetic characterization of hatchery populations of Korean spotted sea bass (Lateolabrax maculatus) using multiplex polymerase chain reaction assays. Genet. Mol. Res. 13(3), 6701-6715 (2014).

22. Shaw, P. W., Turan, C., Wright, J. M., O'Connell, M. \& Carvalho, G. R. Microsatellite DNA analysis of population structure in Atlantic herring (Clupea harengus), with direct comparison to allozyme and mtDNA RFLP analyses. Heredity 83, 490-499 (1999).

23. Shao, C. W., Chen, S. L., Xu, G. B., Liao, X. L. \& Tian, Y. S. Eighteen novel microsatellite markers for the Chinese sea perch, Lateolabrax maculatus. Conserv. Genet. 10, 623-625 (2009).

24. Zhang, H. R., Niu, S. F., Wu, R. X., Zhai, Y. \& Tian, L. T. Development and characterization of 26 polymorphic microsatellite markers in Lateolabrax maculatus and cross-species amplification for the phylogenetically related taxa. Biochem. Syst. Ecol. 66, 326-330 (2016).

25. Zhao, Y. et al. Isolation of microsatellite markers for Lateolabrax japonicus and polymorphic analysis. Zool. Res. 32(5), 515-520 (2011) (In Chinese with English abstract).

26. Botstein, D., White, R. L., Skolnick, M. \& Davis, R. W. Construction of a genetic linkage map in man using restriction fragment length polymorphisms. Am. J. Hum. Genet. 32, 314-331 (1980).

27. Wan, N. W., Rolland, J. L., Bonhomme, F. \& Phongdara, A. Population genetic structure of Penaeus merguiensis, Thailand based on nuclear DNA variation. J. Exp. Mar. Biol. Ecol. 311(1), 63-78 (2004).

28. Xiao, Q. Z. et al. Genetic diversity analysis of wild and cultured megalopa population of Eriocheir sinensis from Yangtze River. Genom. Appl. Biol. 36(5), 1935-1945 (2017) (In Chinese with English abstract).

29. Liu, F. et al. High genetic diversity and substantial population differentiation in grass carp (Ctenopharyngodon idella) revealed by microsatellite analysis. Aquaculture 297, 51-56 (2009).

30. Halasz, J., Pedryc, A., Ercisli, S., Yilmaz, K. U. \& Hegedus, A. S-genotyping supports the genetic relationships between Turkish and Hungarian apricot germplasm. J. Am. Soc. Hortic. Sci. 135(5), 410-417 (2010).

31. Wang, S. H., Zhang, C., Shang, M., Wu, X. G. \& Cheng, Y. X. Genetic diversity and population structure of native mitten crab (Eriocheir sensu stricto) by microsatellite markers and mitochondrial COI gene sequence. Gene 693, 101-113 (2019).

32. Li, M. Y., Zhao, M. Z., Zhong, A. H. \& Xue, L. Y. Comparative analysis of genetic variation by isozymes between two stocks of the sea Bass (Lateolabrax japonicus) from Rizhao \& Xiamen sea areas, China. J. Zhejiang Ocean Univ. Nat. Sci. 22(2), 121-124 (2003) (In Chinese with English abstract).

33. Li, M. Y., Zhao, M. Z., Zhong, A. H. \& Xue, L. Y. Comparative analysis of RAPD genetic variation between two stocks of sea bass (Lateolabrax aponicus) from Shandong Rizhao and Fujian Xiamen sea areas. Oceanol. Limnol. Sin. 34(6), 618-624 (2003) (In Chinese with English abstract).

34. Machado-Schiaffino, G. \& Garcia-Vazquez, E. Isolation and characterization of microsatellite loci in Merluccius australis and cross-species amplification. Mol. Ecol. Resour. 9(2), 585-587 (2009).

35. Freeland, J. R. Molecular ecology 112-116 (Wiley, Chichester, 2005).

36. Li, D. Y., Yin, Q. Q., Hou, N., Sun, X. W. \& Liang, L. Q. Genetic diversity of different ecologo-geographical populations of yellow catfish Pelteobagrus eupogon. Oceanol. Limnol. Sin. 40(4), 460-469 (2009) (In Chinese with English abstract).

37. Shoji, J. \& Tanaka, M. Influence of spring river flow on the recruitment of Japanese sea perch Lateolabrax japonicus into the Chikugo estuary, Japan. . Sci. Mar. 70(2), 159-164 (2006).

38. Liu, B., Kuang, Y. Y., Tong, G. X. \& Yin, J. S. Analysis of genetic diversity on 9 wild stocks of Taimen (Hucho taimen) by microsatellite markers. Zool. Res. 32(6), 597-604 (2011) (In Chinese with English abstract).

39. Clegg, S. M. et al. Genetic consequences of sequential founder events by an island-colonizing bird. Proc. Natl. Acad. Sci. USA 99, 8127-8132 (2002).

40. Pruett, C. L. \& Winker, K. Northwestern song sparrow populations show genetic effects of sequential colonization. Mol. Ecol. 14, 1421-1434 (2005).

41. An, H. S. et al. Population genetic structure of the sea bass (Lateolabrax japonicus) in Korea based on multiplex PCR assays with 12 polymorphic microsatellite markers. Genes Genom. 36, 247-259 (2014).

42. Van Oosterhout, C., Hutchinson, W. F., Willis, D. P. M. \& Shipley, P. F. Microchecker: Software for identifying and correcting genotyping errors in microsatellite data. Mol. Ecol. Notes 4, 535-538 (2004).

43. Dempster, A. P., Laird, N. M. \& Rubin, D. B. Maximum likelihood from incomplete data via the EM algorithm. J. R. Stat. Soc. B 39, 1-38 (1977).

44. Raymond, M. \& Rousset, F. GENEPOP (version 12): Population genetics software for exact tests and ecumenicism. J. Hered. 86, 248-249 (1995).

45. Excoffier, L. \& Lischer, H. E. L. Arlequin suite ver 3.5: A new series of programs to perform population genetics analyses under Linux and windows. Mol. Ecol. Resour. 10, 564-567 (2010).

46. Wang, S. H. et al. Identification and genetic diversity analysis of Chinese mitten crab (Eriocheir sinensis) in the Liao river area. J. Northeast. Agric. Univ. 25, 43-53 (2018).

47. Tamura, K. et al. MEGA 5: Molecular evolutionary genetics analysis using maximum likelihood, evolutionary distance, and maximum parsimony methods. Mol. Biol. Evol. 28, 2731-2739 (2011).

48. Tamura, K. \& Nei, M. Estimation of the number of nucleotide substitutions in the control region of mitochondrial DNA in humans and chimpanzees. Mol. Biol. Evol. 10, 512-526 (1993). 
49. Maruyama, T. \& Fuerst, P. A. Population bottlenecks and nonequilibrium models in population genetics. I. Allele numbers when populations evolve from zero variability. Genetics 111, 675-689 (1985).

50. Pritchard, J. K., Stephens, M. \& Donnelly, P. Inference of population structure using multi locus genotype data. Genetics 155, 945-959 (2000).

51. Jombart, T. Adegenet: An R package for the multivariate analysis of genetic markers. Bioinformatics 24, 1403-1405 (2008).

\section{Acknowledgements}

This research was supported by the Basic Research Fund for National Non-Profit Institutes (2015M03), Shanghai Natural Science Fund Project (18ZR1449800), Central Public-interest Scientific Institution Basal Research Fund, CAFS ( NO.2021JC0102) and the National Infrastructure of Fishery Germplasm Resources.

\section{Author contributions}

W.W. and M.L. designed the study; C.W., L.Z., M.C and Z.H collected the animals; W.W. and Z.M. carried out the experiment; W.W., M.C and Z.F. performed data analysis; W.W., O.L., F.Y. and J.K. wrote the manuscript. All authors reviewed the manuscript.

\section{Competing interests}

The authors declare no competing interests.

Additional information

Correspondence and requests for materials should be addressed to L.M.

Reprints and permissions information is available at www.nature.com/reprints.

Publisher's note Springer Nature remains neutral with regard to jurisdictional claims in published maps and institutional affiliations.

(c) (i) Open Access This article is licensed under a Creative Commons Attribution 4.0 International License, which permits use, sharing, adaptation, distribution and reproduction in any medium or format, as long as you give appropriate credit to the original author(s) and the source, provide a link to the Creative Commons licence, and indicate if changes were made. The images or other third party material in this article are included in the article's Creative Commons licence, unless indicated otherwise in a credit line to the material. If material is not included in the article's Creative Commons licence and your intended use is not permitted by statutory regulation or exceeds the permitted use, you will need to obtain permission directly from the copyright holder. To view a copy of this licence, visit http://creativecommons.org/licenses/by/4.0/.

(c) The Author(s) 2021 